\title{
Erste Ergebnisse des deutschen kinder- und jugendpsychiatrischen Anorexieregisters
}

\author{
Nachwuchspreis der Zeitschrift für Kinder-und \\ Jugendpsychiatrie und Psychotherapie für \\ Frau Dr. med. Katharina Bühren und Dr. med. Manuel Föcker
}

Martin Holtmann

LWL-Universitätsklinik Hamm der Ruhr-Universität Bochum, Klinik für Kinder- und Jugendpsychiatrie, Psychotherapie und Psychosomatik, Bochum

Die wissenschaftliche Redaktion der Zeitschrift für Kinderund Jugendpsychiatrie und Psychotherapie hatte im vergangenen Jahr zum zweiten Mal alle Autorinnen und Autoren, die bei der Einreichung ihrer Arbeit jünger als 40 Jahre alt waren, eingeladen, sich um den Preis für das beste Paper zu bewerben. Es konnten sowohl Originalarbeiten als auch Übersichtsarbeiten, die im Jahre 2017 in der Zeitschrift veröffentlich wurden, eingereicht werden. Das Preisgeld von $500,-€$ für diesen Nachwuchspreis wurde dankenswerterweise erneut vom Hogrefe Verlag zur Verfügung gestellt.

Hintergrund der Idee zu diesem Nachwuchspreis ist das Anliegen, die Zeitschrift noch mehr in das Bewusstsein der jüngeren forschenden Kolleginnen und Kollegen zu rücken und die Zeitschrift weiter als Instrument zur Förderung des wissenschaftlichen Nachwuchses zu stärken. Zugleich ergänzt der Preis eine Reihe von Maßnahmen, die dazu dienen sollen, dass die Zeitschrift als Publikationsort attraktiv bleibt: Dazu gehören die schnelle Onlineverfügbarkeit angenommener Arbeiten innerhalb weniger Wochen (Advance Articles/online first) mit entsprechend früher Sichtbarkeit u.a. bei PubMed und der unentgeltliche Zugang für englische Arbeiten (open access).

In diesem Jahr haben die Herausgeberinnen und Herausgeber der Zeitschrift für Kinder- und Jugendpsychiatrie und Psychotherapie entschieden, den Preis für das beste Nachwuchspaper zu gleichen Teilen an Frau Dr. med. Katharina Bühren und Herrn Dr. med. Manuel Föcker zu verleihen für ihre englischsprachige Originalarbeit „First Sociodemogra- phic, Pretreatment and Clinical Data from a German WebBased Registry for Child and Adolescent Anorexia Nervosa" (Bühren et al., 2017). Die Preisverleihung erfolgte auf der Forschungstagung der Deutschen Gesellschaft für Kinder- und Jugendpsychiatrie, Psychosomatik und Psychotherapie (DGKJP) in Tübingen im März 2018.

Die Herausgeberinnen und Herausgeber zeichnen die Preisträgerin und den Preisträger aus zwei Gründen aus: zum einen natürlich für die Originalarbeit selbst, die die ersten Daten aus dem internetbasierten deutschen Register für Anorexia nervosa bei Kindern und Jugendlichen in Deutschland darstellt und diskutiert. Zum anderen würdigen sie aber auch die Arbeit in einem umfassenderen Sinn, nämlich die buchstäblich jahrelange Vorarbeit für den Aufbau des Anorexieregisters. An ihr hatten Frau Dr. Bühren und Herr Dr. Föcker maßgeblichen Anteil. Nach geduldiger Bewältigung von ethischen, datenschutzrechtlichen, finanziellen und praktischen Hürden ist es gelungen, deutschlandweit an 12 Universitätskliniken und 2 Versorgungskliniken mit kinder- und jugendpsychiatrischen Abteilungen eine gemeinsame Datenbank zu errichten, für die seit Januar 2015 sukzessive Patientinnen und Patienten rekrutiert werden konnten. Neben der Preisträgerin und dem Preisträger waren hieran eine Vielzahl von Kolleginnen und Kollegen der 14 Kliniken und des Instituts für Medizinische Informatik der Universität Kiel beteiligt, die auch als Koautorinnen und Koautoren der Arbeit firmieren. Unterstützt wird das Register vom Central Information Office 
in Marburg, das als Nachfolger der ehemaligen IT Koordination des Kompetenznetzes Parkinson die informationstechnische Plattform für das Register entwickelt hat.

Frau Dr. Bühren als Erstautorin, Herrn Dr. Föcker als Letztautor und ihren Koautorinnen und Koautoren ist es gelungen, soziodemografische und klinische Daten von 258 Patientinnen und Patienten zu erfassen und auszuwerten, die aufgrund einer Anorexia nervosa stationär behandelt wurden. Zusätzlich wurden Art und Ausmaß der ambulanten Vorbehandlung erhoben. Neben wichtigen Ergebnissen zur Ausprägung der Essstörung bei Aufnahme und Entlassung, der Altersstruktur der Patientinnen und Patienten und ihrem im Vergleich zur deutschen Normalbevölkerung im Mittel höheren Bildungsniveau geben die Analysen einen Einblick in die Zugangswege zur stationären Behandlung. Trotz einiger Einschränkungen geben die Daten zur Vorbehandlung Hinweise auf möglicherweise vermeidbare Verzögerungen und Versorgungslücken. So scheint das Wissen über Essstörungen in der primärärztlichen Versorgung in Deutschland noch nicht ausreichend zu sein, Wartezeiten auf stationäre und teilstationäre Behandlungen sind zu lang, und der Übergang zwischen den Behandlungssektoren gelingt nicht immer zufriedenstellend. Die Autorinnen und Autoren diskutieren, ob ein Ausbau spezialisierter ambulanter Angebote für Kinder und Jugendliche mit Essstörungen zu einem verbesserten Behandlungsergebnis führen könnte. Ähnliches konnte in Großbritannien bereits gezeigt werden.

Allen Autorinnen und Autoren der Arbeit ist gemeinsam etwas gelungen, das oft gefordert und nur selten erfolgreich umgesetzt wird, nämlich die Nutzbarmachung von klinischen Routinedaten für die Forschung mit dem Ziel, die Forschungsergebnisse dann wieder für die Versorgung fruchtbar zu machen. Dem Anorexieregister, das mittlerweile 16 Kliniken umfasst, ist zu wünschen, dass auf diese erste grundlegende Arbeit weitere Studien folgen, die zu einer noch besseren Qualität der Versorgung von Kindern und Jugendlichen mit Anorexia nervosa beitragen.
Auch im laufenden Jahr stellt der Hogrefe Verlag wieder ein Preisgeld von 500,- $€$ für die Verleihung des Nachwuchspreises zur Verfügung. Die Herausgeberinnen und Herausgeber der Zeitschrift für Kinder- und Jugendpsychiatrie und Psychotherapie sind dem Verlag dafür sehr dankbar und laden erneut alle Erst- und Letztautorinnen und -autoren ein, die bei Einreichung ihrer Arbeit jünger als 40 Jahre alt sind, sich um den Preis für das beste Paper, das im Jahre 2018 veröffentlicht wurde/wird, zu bewerben. Die Preisverleihung erfolgt im Frühjahr 2019 auf dem XXXVI. DGKJP-Kongress, der vom 10. bis 13. April 2019 in Mannheim stattfindet. Bewerbungen können noch bis zum Jahresende unter Angabe der Adresse und des Geburtsdatums beim geschäftsführenden Herausgeber erfolgen.

\section{Literatur}

Bühren, K., Herpertz-Dahlmann, B., Dempfle, A., Becker, K., Egberts, K. M., Ehrlich, S., Fleischhaker, C., von Gontard, A., Hahn, F., Jaite, C., Kaess, M., Legenbauer, T., Renner, T.J., Schrötter, E., Schulze, U., Sinzig, J., Antony, G., Hebebrand, J., Föcker, M. (2017). First Sociodemographic, Pretreatment and Clinical Data from a German Web-Based Registry for Child and Adolescent Anorexia Nervosa. Zeitschrift für Kinder- und Jugendpsychiatrie und Psychotherapie, 45, 393-400. https://doi.org/10.1024/1422-4917/ a000544

Adresse für Bewerbungen um den Nachwuchspreis für das beste Paper 2018 .

\section{Prof. Dr. Dr. Martin Holtmann}

LWL-Universitätsklinik Hamm der Ruhr-Universität Bochum Klinik für Kinder- und Jugendpsychiatrie, Psychotherapie und Psychosomatik

Heithofer Allee 64

59071 Hamm

Deutschland

martin.holtmann@lwl.org 